\title{
Dielectric properties of thin insulating layers measured by Electrostatic Force Microscopy
}

\author{
C. Riedel ${ }^{1,2,3}$, R. Arinero ${ }^{1, a}$, Ph. Tordjeman $^{4}$, M. Ramonda ${ }^{5}$, G. Lévêque ${ }^{1}$, G.A. Schwartz ${ }^{6}$, D.G. de Oteyza ${ }^{2}$, \\ A. Alegría ${ }^{3,6}$, and J. Colmenero ${ }^{2,3,6}$ \\ 1 Institut d'Électronique du Sud (IES), UMR CNRS 5214, Université Montpellier II, CC 082, Place E. Bataillon, \\ 34095 Montpellier Cedex, France \\ 2 Donostia International Physics Center, Paseo Manuel de Lardizabal 4, 20018 San Sebastián, Spain \\ 3 Departamento de Física de Materiales UPV/EHU, Facultad de Química, Apartado 1072, 20080 San Sebastián, Spain \\ 4 Université de Toulouse, INPT - CNRS, Institut de Mécanique des Fluides (IMFT), 1 allée du Professeur Camille Soula, \\ 31400 Toulouse, France \\ ${ }^{5}$ Laboratoire de Microscopie en Champ Proche (LMCP), Centre de Technologie de Montpellier, Université Montpellier II, \\ CC 082, Place E. Bataillon, 34095 Montpellier Cedex, France \\ ${ }^{6}$ Centro de Física de Materiales (CSIC-UPV/EHU), Materials Physics Center MPC, Edificio Korta, 20018 San Sebastián, \\ Spain
}

Received: 25 September 2009 / Received in final form: 4 December 2009 / Accepted: 16 December 2009 Published online: 26 February 2010 - (c) EDP Sciences

\begin{abstract}
In order to measure the dielectric permittivity of thin insulting layers, we developed a method based on electrostatic force microscopy (EFM) experiments coupled with numerical simulations. This method allows to characterize the dielectric properties of materials without any restrictions of film thickness, tip radius and tip-sample distance. The EFM experiments consist in the detection of the electric force gradient by means of a double pass method. The numerical simulations, based on the equivalent charge method (ECM), model the electric force gradient between an EFM tip and a sample, and thus, determine from the EFM experiments the relative dielectric permittivity by an inverse approach. This method was validated on a thin $\mathrm{SiO}_{2}$ sample and was used to characterize the dielectric permittivity of ultrathin poly(vinyl acetate) and polystyrene films at two temperatures.
\end{abstract}

\section{Introduction}

Physical study of complex materials as nano-structured materials and self-assembly polymers requires the development of methods to characterize their properties at the nano and microscale. Particularly, nano-characterization of dielectric properties presents a great interest to understand the behaviour of these complex systems under electromagnetic radiation and to study their dynamics at the nanoscale, in bulk or in confined geometry. We present here a method to measure the dielectric properties of thin insulating films. This method is based on electrostatic force microscopy (EFM) experiments coupled with numerical simulations and provide quantitative measurements of the relative dielectric permittivity, $\varepsilon_{r}$, of complex materials in the liquid or solid state.

In typical EFM experiments, dc or ac bias voltages are applied between the tip and the sample via a conductive cantilever. EFM is generally used to measure the surface potential (Kelvin probe force atomic microscopy KPFM) on semiconducting materials [1], and to image lo-

\footnotetext{
a e-mail: richard.arinero@ies.univ-montp2.fr
}

calized charges on surfaces [2], dielectric constant variations $[3,4]$ and potentials $[1,5]$. Recently, Crider et al. $[6,7]$ used ultra high vacuum atomic force microscopy (UHV$\mathrm{AFM})$ in order to characterize the complex dielectric permittivity $\left(\varepsilon^{*}(\omega)=\varepsilon^{\prime}-i \varepsilon^{\prime \prime}\right)$ of poly(vinyl acetate) polymer (PVAc). This experiment was realized by applying an ac bias voltage of variable frequency $(\omega)$. From the in and quadrature phase components of the sensor signal response and using a phenomenological model, they obtained the qualitative frequency dependence of $\varepsilon^{\prime}$ and $\varepsilon^{\prime \prime}$. Other reported works were devoted to the determination of the modulus of the local dielectric permittivity without taking into account the possible frequency response of the material. We can mention for instance the works of Krayev et al. $[3,4]$ related to the study of polymers blend in the form of layer of several microns thickness. The authors showed that an electric contrast could be obtained on EFM images and that such a contrast is related to the variations of $\varepsilon_{r}$. They also quantified the value of $\varepsilon_{r}$ in the frame of a simple spherical capacitor model, which is valid for large thickness of the sample in comparison with the tip radius and the tip-sample distance. Moreover, dielectric constants of two reference polymers are 
required to measure a third unknown one. Finally, a different approach has been recently proposed by Fumagalli et al. [8] and Gomila et al. [9]. The authors developed the so-called "Nanoscale Capacitance Microscopy", which is based on high-resolution measurement of capacitancedistance curves. While a sinusoidal voltage is applied between the AFM tip and the bottom electrode of the sample, the ac current is measured using a state of the art high sensitivity current amplifier. From the sample impedance, the tip-sample capacitance can be obtained according to the distance. Then, it is possible to extract the dielectric permittivity of the sample by fitting the capacitancedistance curve with an appropriate model. The authors proposed an analytical model, of which the validity was proven for film thickness lower than $100 \mathrm{~nm}$ [9].

A number of models describing probe-sample interactions have been proposed in the two last decades. Earlier models treated the probe surface as an equipotential with an assumed distribution of charges, such as a single point charge [10] or a uniformly charged line [11], and the probe-sample interaction was approximated as the interaction between the assumed charge distribution and its image with respect to the sample surface. Another group of models introduced geometric approximations to the probe shape and solved the probe-sample capacitance problem either by exactly solving the boundary value problem, e.g., the sphere model [12] and the hyperboloid model [13], or by introducing further approximations to the electric field between the probe and the sample [14-16]. These models provide convenient analytic expressions of the probe-sample interaction; however, more sophisticated models are demanded for studying the lateral variation of the sample surface properties (e.g., topography and trapped charges distribution) or to take into account the presence of a dielectric film of variable thickness. A second family of models, also called equivalent charge method (ECM), replaced the probe and the sample by a series of point charges and/or line charges and their image charges [17-20]. Based on this method, interactions between the probe and a conductive or dielectric sample with topographic and/or dielectric inhomogeneities [21-23] have been studied. This approach was capable of accommodating different scenarios. The third family of approaches used numerical methods such as the finite element method [24], the self-consistent integral equation method [25], and the boundary element method [26]. The main advantage of these models is their ability to take into account the exact geometry of the EFM probe, which permits comparison of different probe tip shapes.

This paper is organized as follow: we present in Section 2, the EFM experiments based on the detection of the electric force gradient by means of a double pass method $[1,27,28]$. Numerical simulations are discussed in Section 3. They were realized in the frame of ECM [17-20] and allow to extract from the EFM experiments, the $\varepsilon_{r}$ value of samples following an inverse approach. This method to measure the dielectric permittivity of insulating layers has been applied to $\mathrm{SiO}_{2}$, material for which the local dielectric permittivity has been already studied in the literature $[8,9]$, and to characterize the dielectric properties of poly(vinyl acetate) (PVAc) and polystyrene (PS) thin films at two temperatures. The experimental results are presented and discussed in Section 4.

\section{EFM experiments}

In order to determine $\varepsilon_{r}$ value of a thin insulating layer, we have developed a EFM method based on the measurement of the electric force gradient $\operatorname{Grad}_{D C} F$ between the tip and the sample holder on which the insulating layer is deposed. The force gradient is related to the cantilevertip-sample capacitance $C(z)$ by $\operatorname{Grad}_{D C} F=\frac{1}{2} \frac{\partial^{2} C(z)}{\partial z^{2}} V_{D C}^{2}$, where $z$ is the tip-sample distance. There are two possibilities to detect the local electrostatic force gradient. The first one is to measure directly the resonance frequency shift $\Delta f_{0}$ keeping the phase shift constant. The second possibility is to measure the mechanical phase shift $\Delta \Phi$ at constant driving frequency. If we consider that the cantilever-tip-sample system can be modelled by a spring mass system, the relationships between frequency $\Delta f_{0}$ or phase shifts $\Delta \Phi$ and force gradient $\operatorname{Grad}_{D C} F$, assuming $\operatorname{Grad}_{D C} F \ll k_{c}$ and $\tan \Delta \Phi \cong \Delta \Phi$ (origin at the resonance frequency), can be written as [27]:

$$
\begin{aligned}
\frac{\Delta f_{0}}{f_{0}} \cong-\frac{1}{2} \frac{\operatorname{Grad}_{D C} F}{k_{c}} \\
\Delta \Phi \cong-\frac{Q}{k_{c}} \operatorname{Grad}_{D C} F
\end{aligned}
$$

where $k_{c}$ and $Q$ are the stiffness of the cantilever and the quality factor, respectively. As expected from relations (1) and (2) the curves $\Delta f_{0}\left(V_{D C}\right)$ and $\Delta \Phi\left(V_{D C}\right)$ have a parabolic shape, $-a_{\Delta f_{0}}(z) V_{D C}^{2}$ and $-a_{\Delta \Phi}(z) V_{D C}^{2}$, where $a_{\Delta f_{0}}(z)$ and $a_{\Delta \Phi}(z)$ are related to the tip-sample capacitance by the expressions:

$$
\begin{aligned}
& a_{\Delta f_{0}}(z)=\frac{f_{0}}{4 k_{c}} \frac{\partial^{2} C(z)}{\partial z^{2}} \\
& a_{\Delta \Phi}(z)=\frac{Q}{2 k_{c}} \frac{\partial^{2} C(z)}{\partial z^{2}} .
\end{aligned}
$$

We point out that although the force gradient can be detected either by measuring the frequency shifts or by measuring the phase shifts, relation (2) is valid only at low dc voltages (for which the approximation $\tan \Delta \Phi \cong \Delta \Phi$ is satisfied) whereas relation (1) is always valid. For high dc voltages the measured phase shift is saturated and does not exhibit a parabolic shape any more. That is why we chose to measure the frequency shift.

Considering the tip as a cone of angle $\theta_{0}$ with a spherical apex of radius $R$ fixed to the extremity of the cantilever, the total capacitance $C(z)$ is a sum of the apex capacitance $C_{\text {apex }}(z)$, i.e. the local capacitance, and the stray capacitance $C_{\text {stray }}(z)$, relative to the tip cone and the cantilever. In their work, Fumagalli et al. [8] have shown that the stray capacitance obeys to a linear relation, $C_{\text {stray }}(z)=-b \Delta z$, and does not contribute to the 


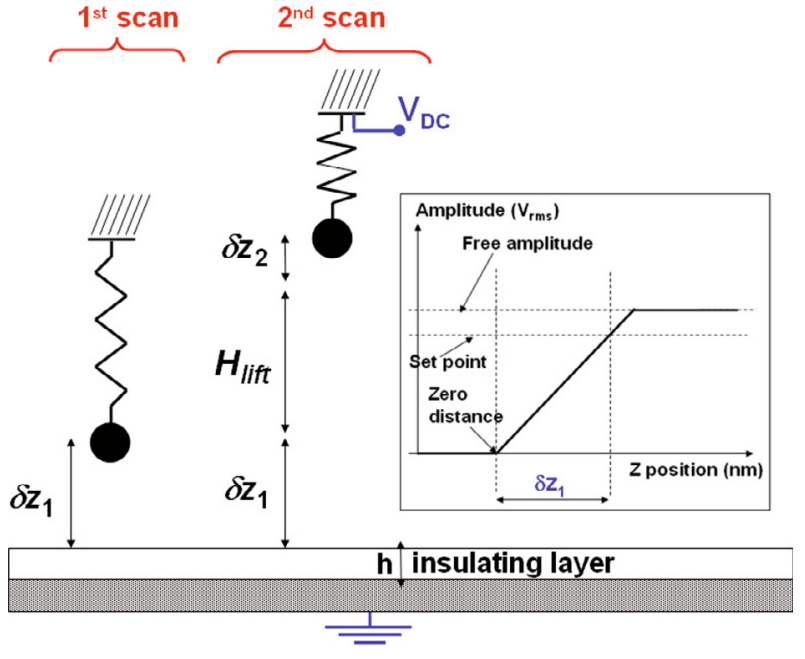

Fig. 1. (Color online) Principle of EFM microscopy using a double pass method. During the first scan topography is acquired. The tip is then retracted by a constant height $H_{\text {lift }}$ and amplitude is reduced by a factor 3. During the second scan, a constant potential is applied on the tip and the dc force gradient is analysed. Inset: typical amplitude-distance curve recorded on a stiff sample. The first scan amplitude $\delta z_{1}$ corresponds to the difference between the $z$-position allowing to reach the set point amplitude and the zero distance.

second derivative of the capacitance $\partial^{2} C(z) / \partial z^{2}$ in the expressions (3) and (4).

The experimental protocol was developed on one single surface position on the basis of a "double pass method" $[1,27,28]$ and the measurement of $a_{\Delta f_{0}}(z)$ parabolic coefficient from the experimental curves $\Delta f_{0}\left(V_{D C}\right)$. EFM experiments are made in ambient air atmosphere and at different temperatures $\left(22{ }^{\circ} \mathrm{C}\right.$ and $\left.70{ }^{\circ} \mathrm{C}\right)$ in the amplitude-controlled mode (Tapping ${ }^{\circledR}$ ). During the first scan the topography is acquired. The tip is then retracted from the surface morphology by a constant height $H_{\text {lift }}$, also called "lift height", and the amplitude of the tip vibration $\delta z$ is reduced in order to stay in the linear regime (amplitude «tip-sample distance). During the second scan, while a potential $V_{D C}$ is applied to the tip (with the sample holder grounded) the electric force gradient $\operatorname{Grad}_{D C} F$ is detected. As shown in Figure 1, during the first scan, the average tip-sample distance $z_{1}$ is approximately equal to the oscillation amplitude $\left(z_{1} \cong \delta z_{1}\right)$. During the second scan, the distance is the sum of the first scan amplitude $\delta z_{1}$ and the lift height $H_{\text {lift }}\left(z_{2} \cong \delta z_{1}+H_{\text {lift }}\right)$ and the cantilever oscillates with an amplitude of $\delta z_{2}$.

The EFM experiments are performed in three steps: first, in order to determine the actual value of the tip radius $R$, we measure $\Delta f_{0}\left(V_{D C}\right)$ curves at several lift height $H_{\text {lift }}$ for a conductive sample. A parabolic fit gets the experimental coefficients $a_{\Delta f_{0}}(z)$ according to the real tip-sample distance. A value of the radius $R$ is then obtained by fitting the $a_{\Delta f_{0}}(z)$ curve with expression (3) in which the tip-sample capacitance is calculated using the equivalent charge model (ECM) (see Section 3). Second, the experiment is performed with a thin insulating layer of the material under study deposited on the conductive substrate. $\Delta f_{0}\left(V_{D C}\right)$ curves are recorded at different lift heights $H_{\text {lift }}$ and are analysed in order to extract experimental coefficients $a_{\Delta f_{0}}(z)$ for each lift height. Once $R$ and $h$, the thickness of the sample measured by AFM, are known from previous experiments, we can fit the $a_{\Delta f_{0}}(z)$ curve using expression (3) in which the capacitance is calculated by ECM, and thereby we obtain the value of the dielectric permittivity $\varepsilon_{r}$. Finally, in a third step, we record an oscillation amplitude-distance curve to quantify the actual values of $\delta z_{1}$ and $z_{2}$ in the previous force gradient experiments. One can note that the measurement of an amplitude-distance curve can damage the tip and should be realized at the end. A typical curve is shown in the inset of Figure 1; the slope of this curve gives the correspondence between the photodetector rms voltage and the real oscillation amplitude. Indeed, if there is no indentation of the tip into the sample, we can consider that amplitude is coarsely equivalent to the distance. The zero distance corresponds to the point where amplitude becomes null. The tip-sample distance is calculated as the difference between the $z$-position of the actuator corresponding to the amplitude set point and the $z$-position corresponding to the zero distance [29].

\section{ECM numerical simulations}

In this section, we show how the tip radius, the tip-sample force, force gradient and capacitance can be calculated using the equivalent charge method (ECM). The advantage of numerical simulation compared to other analytical expression is that the calculated force is exact and allows to work without any restriction about the thickness of the insulating film, the tip radius and the tip-sample distance. We will first consider the case of a tip in front of a metallic plate, and then we will deduce the force and the force gradient for a system composed by a tip in front of a dielectric layer over a metallic plate.

The case of a system composed by a tip in front of a conductive plane has been treated by Belaidi et al. [18]. The idea of ECM is to find a discrete charge distribution ( $N_{C}$ charge points $q_{i}$ at a distance $z_{i}$ on the axis $x=0$ ) that will create a given potential $V$ at the tip surface. The tip geometry is represented by an half of sphere of radius $R$ surmounted by a cone with a characteristic angle $\theta_{0}=30^{\circ}$. The conductive plane at a zero potential is created by the introduction of an electrostatic image tip with $-q_{i}$ charges at a distance $-z_{i}$ on the $z$-axis (Fig. 2). The value of the charges $q_{i}$ is fixed in such way that the $M$ potential $V_{n}$, with $n=1, \ldots, M$, calculated at test point $n$ at the tip surface are equal to $V$. If we introduce $D_{i, n}=1 / d_{i, n}-$ $1 / d_{i, n}^{*}$ (where $d_{i, n}$ and $d_{i, n}^{*}$ are the distances between the point $n$ and the effective and image charge $i$, respectively) we can express the potential $V_{n}$ as:

$$
V_{n}=\sum_{i}^{N_{c}} \frac{D_{i, n} q_{i}}{4 \pi \varepsilon_{0}} .
$$




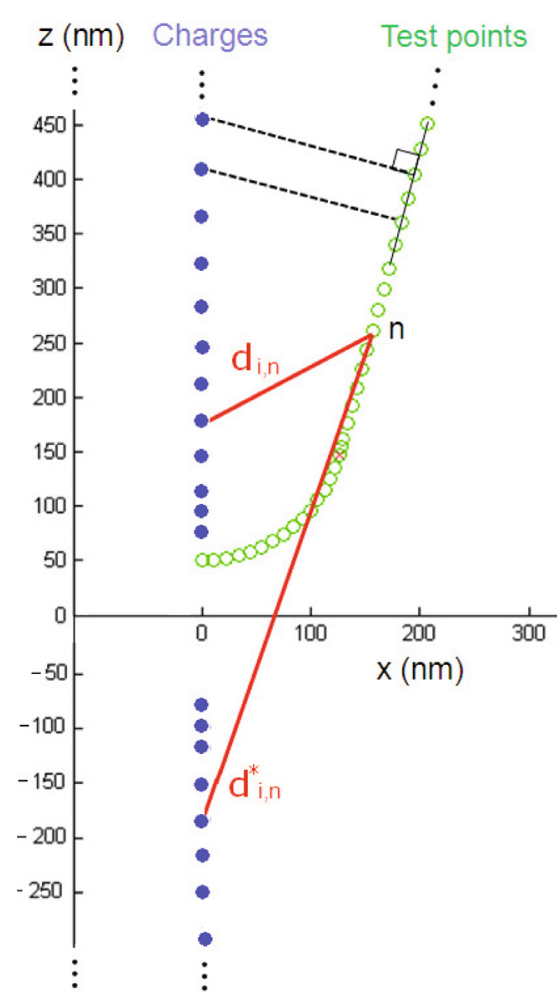

Fig. 2. (Color online) Representation of the charges $(\bullet z>0)$, image charges $(\bullet z<0)$, and test points (o) modelling the tip. Charges simulating the apex have to been place manually to reproduce the strong curvature of the equipotential.

The best value of $q_{i}$ is obtained using the least mean square method:

$$
\frac{\partial}{\partial q_{i}} \sum_{n}^{M}\left(V_{n}-V\right)^{2}=0 .
$$

Expliciting the derivative of the potential, the system to solve becomes:

$$
\sum_{n}^{M}\left(\sum_{i}^{N_{c}} \frac{D_{i, n} q_{i}}{4 \pi \varepsilon_{0}}-V\right) \frac{D_{i, n}}{4 \pi \varepsilon_{0}}=0 .
$$

Then, knowing the charge and image charge distributions, the total electrostatic force acting on the tip and the tip-sample capacitance can be calculated. The coefficient $a_{\Delta f_{0}}(z)$ (or $\left.a_{\Delta \Phi}(z)\right)$ is also obtained according to equation (3) (or Eq. (4)). As shown in Figure 3, this coefficient is very sensitive to the tip radius $(R)$. Following an inverse approach, it is possible to determine the $R$ value from the experimental curve $a_{\Delta f_{0}}(z)$ (or $a_{\Delta \Phi}(z)$ ).

When the system is composed by a tip in front of a dielectric layer on a conductive substrate, simulations are more complex. This problem has been treated by Sacha et al. [19] introducing the Green function formalism and also by Durand [20]. We consider one charge $q_{i}$ in the air at a distance $z_{i}$ of a dielectric layer of thickness $h$ and of dielectric constant $\varepsilon_{r}$. The insulating layer is placed over a conductive substrate. $V_{0}^{i}$ and $V_{1}^{i}$ are respectively the

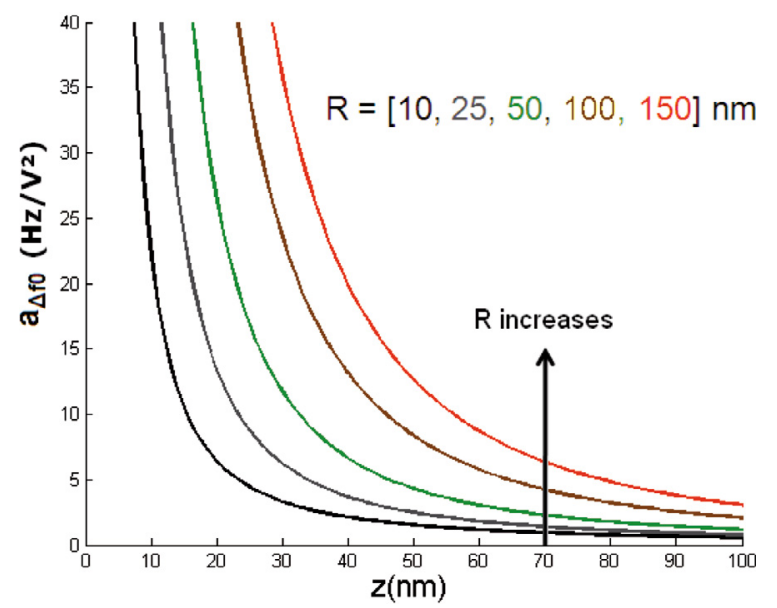

Fig. 3. (Color online) Tip radius effects on $a_{\Delta f 0}(z)$ over a conductive plate. $a_{\Delta f_{0}}(z)$ increases when the tip radius increases.

potentials created by the charge $q_{i}$ in the air and in the dielectric. In order to satisfy the limit conditions $\left(V_{0}^{i}=V_{1}^{i}\right.$ and $\varepsilon_{0} \frac{\partial V_{0}^{i}}{\partial z}=\varepsilon_{0} \varepsilon_{r} \frac{\partial V_{1}^{i}}{\partial z}$ at the air/dielectric interface, and, $V_{1}^{i}=0$ at the dielectric/substrate interface), we introduce two series of image charges, one created in the conductive substrate and one in the air.

The equivalent potential calculated by ECM in the air results from the source, its image in the dielectric and the infinite series of image charges in the conductive substrate. One can introduce the "reciprocal distance", $D+$, between a point of coordinate $(\rho, z)$ and the charge $q_{i}$ (id. its image $\left.(D-), D \pm=1 / \sqrt{\rho^{2}+\left(z \mp z_{i}\right)^{2}}\right)$, and the reciprocal distance $A$ corresponding to the infinite series of image $\left(A=\sum_{n=0}^{\infty} k^{n} / \sqrt{\rho^{2}+\left(z+2(n+1) h+z_{i}\right)^{2}}\right.$, where the constant $\left.k=-\frac{\varepsilon_{r}-1}{\varepsilon_{r}+1}\right)$. Then, the potential $V_{0}^{i}$ created in the air by one charge $q_{i}$ is expressed as:

$$
V_{0}^{i}=\frac{q_{i}}{4 \pi \varepsilon_{0}}\left(D_{+}+k D_{-}-\left(1-k^{2}\right) A\right) .
$$

The potential $V_{1}^{i}$ created in the dielectric is the sum of the two infinite series of images. Introducing the reciprocal distance for the images in the conductive substrate, $B\left(B=\sum_{n=0}^{\infty} k^{n} / \sqrt{\rho^{2}+\left(z-2 n h-z_{i}\right)^{2}}\right)$, we obtain:

$$
V_{1}^{i}=\frac{q_{i}}{4 \pi \varepsilon_{0}}(1-k)(B-A) .
$$

The value of each $q_{i}$ is then found by solving equation (7), inserting the potential $V_{0}^{i}$ calculated after equation (9), at each test point representing the tip surface. Knowing the charge and image charge distributions, the total electrostatic force acting on the tip and the tip-sample capacitance can be calculated. The coefficient $a_{\Delta f_{0}}(z)$ (or $a_{\Delta \Phi}(z)$ ) is obtained according to equation (3) (or Eq. (4)). In Figures $4 \mathrm{a}$ and $4 \mathrm{~b}$, we present the repartition of the equipotentials in air and in a dielectric layer $\left(\varepsilon_{r}=4\right)$ for two different thicknesses. Figures 5 and 6 show the effects of $h$ and $\varepsilon_{r}$, on the coefficient $a_{\Delta f_{0}}(z)$. 

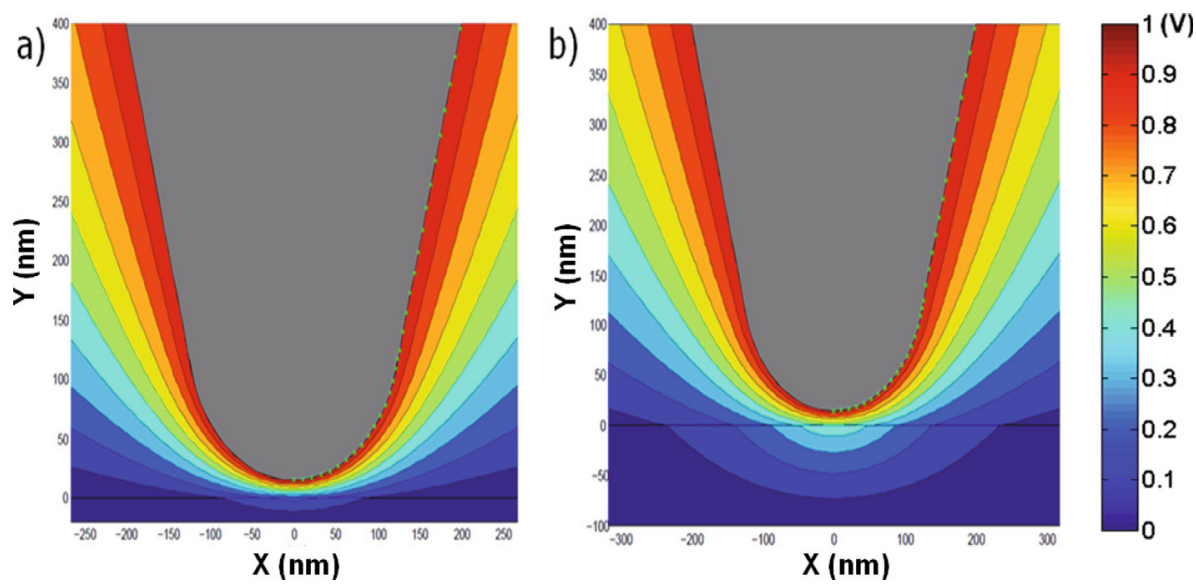

Fig. 4. (Color online) Potential created in the air $(z>0 \mathrm{~nm})$ and in the dielectric $(z<0)$ by a tip $\left(R=130 \mathrm{~nm}, \theta_{0}=30^{\circ}\right)$ in front of a dielectric layer of height of (a) $h=20 \mathrm{~nm}$ and (b) $h=100 \mathrm{~nm}$ with a dielectric constant $\varepsilon_{r}=4$.

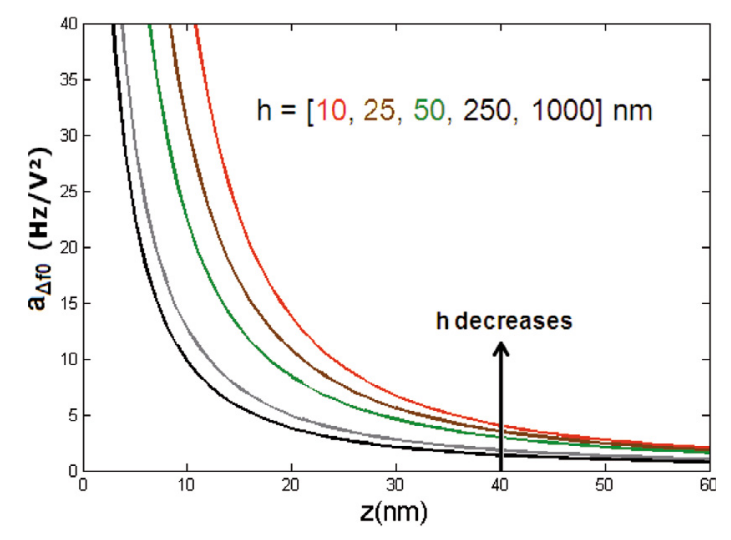

Fig. 5. (Color online) Thickness effects on $a_{\Delta f 0}(z)$ over an insulator with a dielectric constant $\varepsilon_{r}=4 . a_{\Delta f 0}(z)$ increases when the thickness of the insulator decreases.

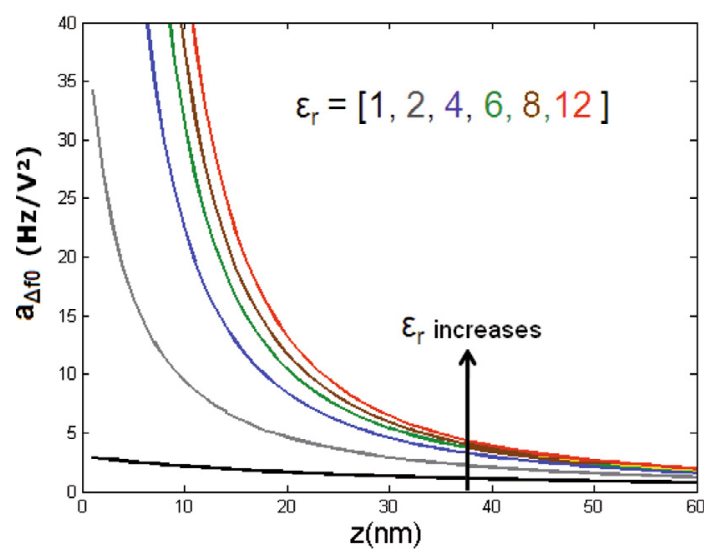

Fig. 6. (Color online) Relative dielectric constant effects on $a_{\Delta f 0}(z)$ over an insulator with a thickness of $40 \mathrm{~nm} . a_{\Delta f 0}(z)$ increases when the dielectric constant increases.

\section{Results and discussion}

We tested our method studying the dielectric constant of an insulating thin layer of $\mathrm{SiO}_{2}$ deposited on a gold substrate (Fig. 7). Our samples were similar to those

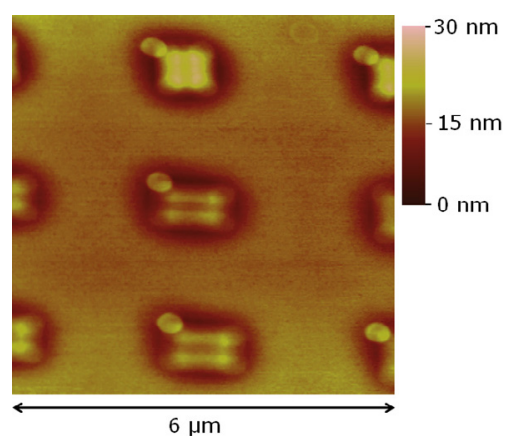

Fig. 7. (Color online) Topography of an insulating thin layer of $\mathrm{SiO}_{2}$ deposited on a gold substrate. The topography is measured by AFM in the amplitude-controlled mode (Tapping ${ }^{\circledR}$ ).

studied by Fumagalli et al. [8]. They were composed of squares of $1 \mu \mathrm{m}$ side deposited by focused ion beam (FIB). FIB (Strata DB235 made by FEI Company) uses a gallium ion beam for localized depositions of distinct materials. The technique allows the deposition of $3 \mathrm{D}$ structures and has a process control precision within a few tens of nanometers $(30 \mathrm{~nm})$. In practice, during the fabrication process some difficulties can be encountered. In Figure 7, we can see some oxide particles deposited very close to the $\mathrm{SiO}_{2}$ squares and the topography is not perfectly homogeneous. The best squares have been selected for our single points EFM measurements. The dark circles correspond to holes created by the beam in the gold substrate layer during the $\mathrm{SiO}_{2}$ deposition. The average thickness of the $\mathrm{SiO}_{2}$ layers measured from the bottom of the holes is approximately $12 \mathrm{~nm}$. We used conductive diamond coated tips (Nanosensors ${ }^{\text {TM }}$ CDT-FMR) having a free oscillating frequency $f_{0}=103 \mathrm{kHz}$ and a stiffness $k_{c}=5.9 \mathrm{~N} \mathrm{~m}^{-1}$. $k_{c}$ was calculated using the socalled thermal tune method [30] based on the thermal noise measurement. The experiments were realized with a Veeco Enviroscope ${ }^{\mathrm{TM}}$ equipped with a Lakeshore temperature controller. In Figure 8, we show the $\Delta f_{0}\left(V_{D C}^{2}\right)$ curve obtained on the gold conductive sample in comparison with the curve obtained on the insulating oxide layer. 


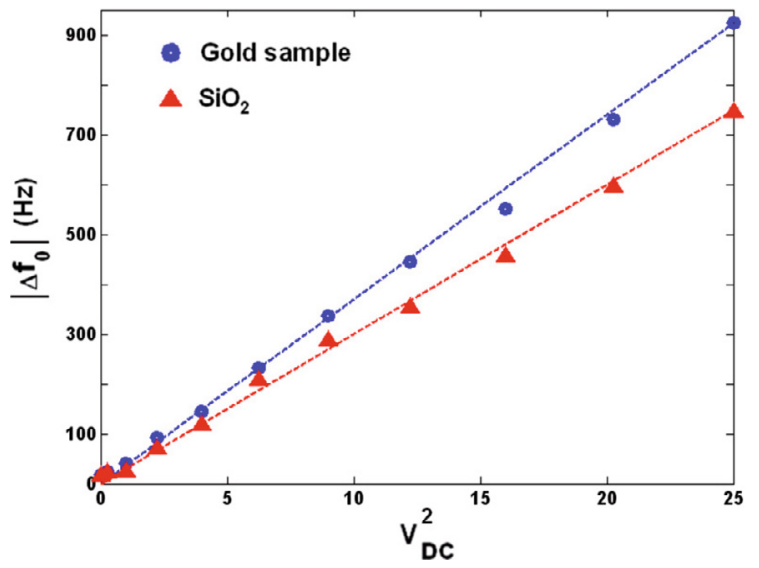

Fig. 8. (Color online) $\Delta f_{0}\left(V_{D C}^{2}\right)$ curves measured on a conductive gold sample $(\bullet)$ and a $\mathrm{SiO}_{2} /$ gold sample $(\boldsymbol{\Lambda})$ with $h_{\mathrm{SiO}_{2}}=$ $12 \mathrm{~nm}$. Both curves were obtained for the same tip-sample distance $z=31 \mathrm{~nm}$. The parabolic fit gives $a_{\Delta f_{0}}(z)=31.7 \mathrm{~Hz} / \mathrm{V}^{2}$ for gold and $a_{\Delta f_{0}}(z)=27.8 \mathrm{~Hz} / \mathrm{V}^{2}$ for $\mathrm{SiO}_{2}$.

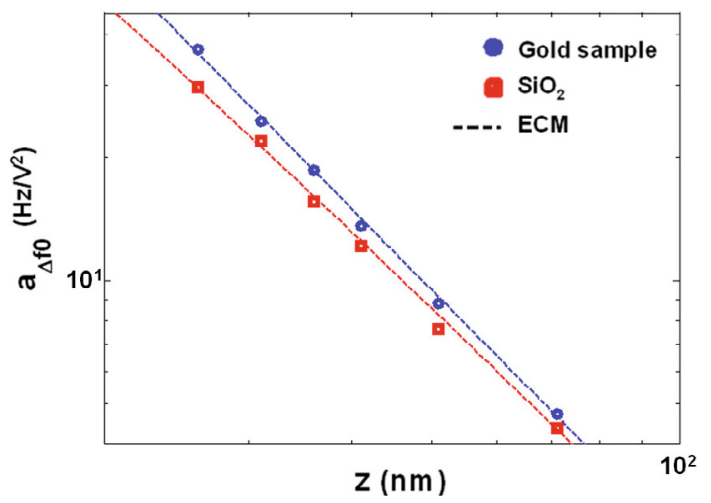

Fig. 9. (Color online) $a_{\Delta f_{0}}(z)$ curves measured on a conductive gold sample $(\bullet)$ and a $\mathrm{SiO}_{2} /$ gold sample $(\boldsymbol{\square})$ with $h_{\mathrm{SiO}_{2}}=12 \mathrm{~nm}$. The tip radius $R=105 \pm 4 \mathrm{~nm}$ is obtained from experiments on gold using ECM. Then, by fitting the $\mathrm{SiO}_{2}$ experiments, we calculated the permittivity of the $\mathrm{SiO}_{2}$ insulating layer: $\varepsilon_{r}=4.5 \pm 1.1$.

Both curves were acquired at the same tip-sample distance $z=31 \mathrm{~nm}$. We observe that the slope of the curve in presence of the oxide layer increases substantially what is revealing a reduction of the local capacitance in accordance with equation (3). By fitting these curves using a parabolic function, we obtained $a_{\Delta f_{0}}=31.7 \mathrm{~Hz} / \mathrm{V}^{2}$ for gold and $a_{\Delta f_{0}}=27.8 \mathrm{~Hz} / \mathrm{V}^{2}$ for $\mathrm{SiO}_{2}$.

In Figure 9, we present the parabolic coefficients $a_{\Delta f_{0}}$ as a function of the real tip-sample distance obtained on gold and $\mathrm{SiO}_{2}$. The fit on gold gives the actual value of tip radius, $R=105 \pm 4 \mathrm{~nm}$ in this case. This value is in good agreement with typical values given by the manufacturer. Then, we calculated the value of the dielectric permittivity of the insulating layer by fitting the points obtained on $\mathrm{SiO}_{2}$. We found $\varepsilon_{r}=4.5 \pm 1.1$ which is in agreement with the value obtained by Fumagalli et al. [8] on the same type of sample. The best-fitting curves were obtained by the least squares method and the final uncertainties were

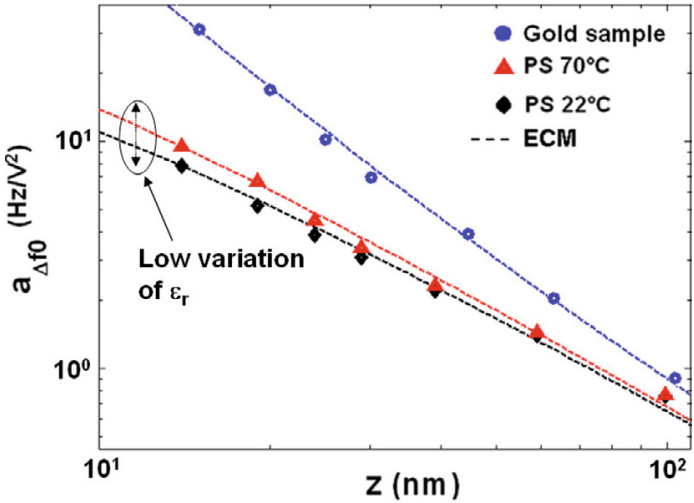

Fig. 10. (Color online) $a_{\Delta f 0}(z)$ curves obtained on a $50 \pm 2 \mathrm{~nm}$ PS thin film at $22{ }^{\circ} \mathrm{C}(\diamond)$ and $70{ }^{\circ} \mathrm{C}(\boldsymbol{\Delta})$ in comparison with the curve obtained on a gold sample $(\bullet)$. The tip radius $R=$ $32 \pm 2 \mathrm{~nm}$ is obtained from experiments on gold using ECM. Fitting PS parabolic coefficients using ECM, we obtained $\varepsilon_{r}=$ $2.2 \pm 0.2$ at $22{ }^{\circ} \mathrm{C}$, and $\varepsilon_{r}=2.6 \pm 0.3$ at $70{ }^{\circ} \mathrm{C}$.

calculated including uncertainties of all others parameters involved in the calculations.

The second serie of experiments was performed on two ultra-thin polymer films. PS $\left(M_{w}=70950 \mathrm{~g} / \mathrm{mol}\right)$ and PVAc $\left(M_{w}=83000 \mathrm{~g} / \mathrm{mol}\right)$ were chosen because both the dielectric strength and its temperature dependence are very different for these two polymers. Additionally, the dielectric responses of both polymers have been previously well characterized in the literature [31-35]. Samples were prepared by spin coating starting from solutions at $1 \%(w / w)$ in toluene. The substrate was composed of a fine gold layer deposited on a glass plate. The small percentage of polymer in solution was selected in order to obtain films with a thickness of about $50 \mathrm{~nm}$ according to reference [36]. We used in this case standard EFM cantilevers (Nanosensors EFM) having a free oscillating frequency $f_{0}=71.42 \mathrm{kHz}$ and a stiffness $k_{c}=4.4 \mathrm{~N} \mathrm{~m}^{-1}$. The experiments were performed on neat PS and PVAc films at room temperature and at $70{ }^{\circ} \mathrm{C}$ (Figs. 10 and 11). The measured thicknesses of the films were $50 \pm 2 \mathrm{~nm}$ for PS and $50 \pm 3 \mathrm{~nm}$ for PVAc at both room temperature and $70{ }^{\circ} \mathrm{C}$. The accuracy of our measurements does not allow detecting any thermal expansion.

The experimental parabolic coefficients $a_{\Delta f_{0}}(z)$ obtained for PS are shown in Figure 10. Measurements at room temperature and at $70{ }^{\circ} \mathrm{C}$ are very close indicating a weak temperature dependence of the dielectric permittivity as expected for this polymer. In addition, there is a big difference between the curve obtained on gold and those obtained on PS. That means that the permittivity of the polymer is rather low. Using the same protocol we obtained the value of the tip radius $R=32 \pm 2 \mathrm{~nm}$ and the dielectric permittivity of PS at $22^{\circ} \mathrm{C}$ and $70{ }^{\circ} \mathrm{C}$ : $\varepsilon_{r}\left(22^{\circ} \mathrm{C}\right)=2.2 \pm 0.2$ and $\varepsilon_{r}\left(70^{\circ} \mathrm{C}\right)=2.6 \pm 0.3$. The experimental parabolic coefficients obtained for PVAc are shown in Figure 11. We can note a significant difference between measurements realized at room temperature and at $70{ }^{\circ} \mathrm{C}$, i.e. below and above the glass transition temperature, $T_{g}$. At $70{ }^{\circ} \mathrm{C}$, the PVAc curve approaches the 


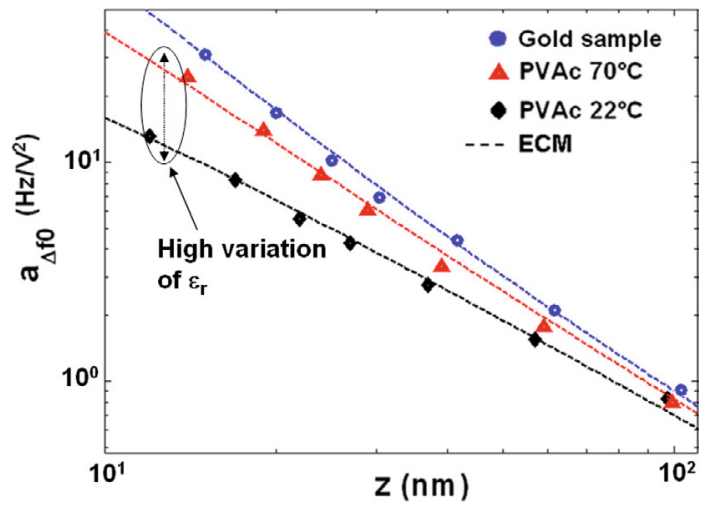

Fig. 11. (Color online) $a_{\Delta f 0}(z)$ curves obtained on a $50 \pm 3 \mathrm{~nm}$ PVAc thin film at $22{ }^{\circ} \mathrm{C}(\boldsymbol{\vee})$ and $70{ }^{\circ} \mathrm{C}(\boldsymbol{\Delta})$ in comparison with the curve obtained on a gold sample $(\bullet)$. The tip radius $R=$ $32 \pm 2 \mathrm{~nm}$ is obtained from experiments on gold using ECM. Fitting PVAc parabolic coefficients using ECM, we obtained $\varepsilon_{r}=2.9 \pm 0.3$ at $22{ }^{\circ} \mathrm{C}$ and $\varepsilon_{r}=8.2 \pm 1.0$ at $70{ }^{\circ} \mathrm{C}$.

gold curve indicating an important increase of $\varepsilon_{r}$. By applying ECM, we obtained $\varepsilon_{r}\left(22{ }^{\circ} \mathrm{C}\right)=2.9 \pm 0.3$ and $\varepsilon_{r}\left(70{ }^{\circ} \mathrm{C}\right)=8.2 \pm 1.0$ for PVAc. The estimated values for PS and PVAc are in good agreement with the macroscopic ones [31-35]. The variation observed in the dielectric permittivity of PVAc is related with its strong dipole moment and the fact that PVAc crossed the glass transition temperature at around $38{ }^{\circ} \mathrm{C}$ increasing the chain mobility and therefore the dielectric permittivity. Opposite, PS has a weak dipole moment and its $T_{g}$ is around $105{ }^{\circ} \mathrm{C}$; therefore, a little or negligible variation of the dielectric permittivity is expected in this case.

We discuss now about performances and limitations of the technique. The theoretical lateral resolution, calculated on the basis of the tip-sample electrostatic interaction $[37,38]$, is given by: $\Delta x=(R z)^{1 / 2}$. Concerning the experiments reported in this paper, if we consider a mean tip-sample distance $z=20 \mathrm{~nm}, \Delta x \cong 45 \mathrm{~nm}$ in the case of the $\mathrm{SiO}_{2}$ sample layer $(R \cong 100 \mathrm{~nm})$, and $\Delta x \cong 25 \mathrm{~nm}$ in the case of the polymer thin films ( $R \cong 30 \mathrm{~nm}$ ). The reached resolutions should be thus good enough to investigate locally the dielectric permittivity of certain nano-structured polymer blends. We focus now on the sensitivity of the technique which can be defined as $\partial a_{\Delta f_{0}} / \partial \varepsilon_{r}$. This quantity can be calculated using ECM and if we analyze the case of the second serie of experiment $(R \cong 30 \mathrm{~nm}$ and $z \cong 20 \mathrm{~nm})$ we obtain, for example, $\partial a_{\Delta f_{0}}\left(\varepsilon_{r}=2\right) / \partial \varepsilon_{r}=2.6 \mathrm{~Hz} / \mathrm{V}^{2}$ and $\partial a_{\Delta f_{0}}\left(\varepsilon_{r}=10\right) / \partial \varepsilon_{r}=0.4 \mathrm{~Hz} / \mathrm{V}^{2}$. The sensitivity clearly decreases when the dielectric permittivity increases. This point can be a limiting factor for the study of highdielectric permittivity materials $\left(\varepsilon_{r}>10\right)$ but not for the study of polymers, for which $2<\varepsilon_{r}<10$.

\section{Conclusions}

We have demonstrated that electrostatic force microscopy (EFM) is a powerful tool to determine quantitatively the dielectric permittivity of an insulating layer. We have detailed an experimental protocol, which consists essentially on determining successively the tip-sample capacitance in the absence and in presence of the sample layer. A quantification of the dielectric permittivity without any experimental restriction has been possible thanks to numerical simulations based on the equivalent charge method (ECM). We believe that numerous applications may potentially be done in a wide range of disciplines. As an example, we showed results on silicon dioxide but also on two different types of polymers (polystyrene and poly(vinyl acetate)) at different temperature. In perspective, this method could be used to characterize and image the local dielectric properties of polymer blends and nanocomposites and study at the nanoscale their molecular dynamics in confined or bulk geometry.

We would like to gratefully acknowledge Pr. J.J. Saenz from Universidad Autónoma de Madrid (MoLE group) and Dr. Gabriel Gomila from the Institute for Bioengineering of Catalonia for fruitful discussions and advices. We are also grateful towards M.J. López Bosque from the "Plataforma de Nanotecnologia" of Barcelona for $\mathrm{SiO}_{2}$ /gold samples preparation. The Donostia Internacional Physics Center (DIPC) financial support is acknowledged. A.A., G.S. and J.C. acknowledge the financial support provided by the Basque Country Government (Ref. No. IT-436-07, Depto. Educación, Universidades e Investigación), the Spanish Ministry of Science and Innovation (Grant No. MAT 2007-63681), the Spanish Ministry of Education (PIE 200860I022), the European Community (SOFTCOMP program) and the PPF Rhéologie et plasticité des matériaux mous hétérogènes 2007-2010, No. 20071656.

\section{References}

1. P. Girard, M. Ramonda, D. Saluel, J. Vac. Sci. Technol. B 20, 1348 (2002)

2. B.D. Terris, J.E. Stern, D. Rugar, H.J. Mamin, Phys. Rev. Lett. 63, 2669 (1989)

3. A.V. Krayev, R.V. Talroze, Polymer 45, 8195 (2004)

4. A.V. Krayev, G.A. Shandryuk, L.N. Grigorov, R.V. Talroze, Macromol. Chem. Phys. 207, 966 (2006)

5. Y. Martin, D.W. Abraham, H.K. Wickramasinghe, Appl. Phys. Lett. 52, 1103 (1988)

6. P.S. Crider, M.R. Majewski, J. Zhang, H. Oukris, N.E. Israeloff, Appl. Phys. Lett. 91, 013102 (2007)

7. P.S. Crider, M.R. Majewski, J. Zhang, H. Oukris, N.E. Israeloff, J. Chem. Phys. 128, 044908 (2008)

8. L. Fumagalli, G. Ferrari, M. Sampietro, G. Gomila, Appl. Phys. Lett. 91, 243110 (2007)

9. G. Gomila, J. Toset, L. Fumagalli, J. Appl. Phys. 104, 024315 (2008)

10. J. Hu, X.D. Xiao, D.F. Ogletree, M. Salmerón, Science 268, 267 (1995)

11. H.W. Hao, A.M. Baró, J.J. Sáenz, J. Vac. Sci. Technol. B 9, 1323 (1991)

12. B.D. Terris, J.E. Stern, D. Rugar, H.J. Mamin, Phys. Rev. Lett. 63, 2669 (1989)

13. L.H. Pan, T.E. Sullivan, V.J. Peridier, P.H. Cutler, N.M. Miskovsky, Appl. Phys. Lett. 65, 2151 (1994) 
14. T. Hochwitz, A.K. Henning, C. Levey, C. Daghlian, J. Slinkman, J. Vac. Sci. Technol. B 14, 457 (1996)

15. S. Hudlet, M. Saint Jean, C. Guthmann, J. Berger, Eur. Phys. J. B 2, 5 (1998)

16. J. Colchero, A. Gil, A.M. Baró, Phys. Rev. B 64, 245403 (2001)

17. G. Mesa, E. Dobado-Fuentes, J.J. Sáenz, J. Appl. Phys. 79, 39 (1996)

18. S. Belaidi, P. Girard, G. Leveque, J. Appl. Phys. 81, 1023 (1997)

19. G.M. Sacha, E. Sahagun, J.J. Saenz, J. Appl. Phys. 101, 024310 (2007)

20. E. Durand, Électrostatique, tome III (Masson, Paris, 1966), p. 233

21. S. Gómez-Moñivas, J.J. Sáenz, Appl. Phys. Lett. 76, 2955 (2000)

22. S. Gómez-Moñivas, L. Froufe-Pérez, A.J. Caamaño, J.J. Sáenz, Appl. Phys. Lett. 79, 4048 (2001)

23. G.M. Sacha, C. Gómez-Navarro, J.J. Sáenz, J. GómezHerrero, Appl. Phys. Lett. 89, 173122 (2006)

24. S. Belaidi, E. Lebon, P. Girard, G. Leveque, S. Pagano, Appl. Phys. A 66, S239 (1998)

25. Z.Y. Li, B.Y. Gu, G.Z. Yang, Phys. Rev. B 57, 9225 (1998)

26. E. Strassburg, A. Boag, Y. Rosenwaks, Rev. Sci. Instrum. 76, $083705(2005)$

27. L. Manzon, P. Girard, R. Arinero, M. Ramonda, Rev. Sci. Instrum. 77, 096101 (2006)

28. L. Portes, M. Ramonda, R. Arinero, P. Girard, Ultramicroscopy 107, 1027 (2007)
29. During the record of the amplitude-distance curve, the tip can be destroyed. We thus recommend to do it at the end of the experiments. Consequently, the adjustable parameter is the lift height. It can vary from positive to negative values, the minimum value corresponding to the height where the tip is in the contact with the sample. In order to maintain the oscillation of the cantilever in a linear regime, we advise to choose a second scan amplitude of approximately 3 or 4 times smaller than $\delta z_{1}$, so $\delta z_{2} \approx 6 \mathrm{~nm}$

30. J.L. Hutter, J. Bechhoefer, Rev. Sci. Instrum. 64, 1868 (1993)

31. G.A. Schwartz, E. Tellechea, J. Colmenero, A. Alegría, J. Non-Cryst. Solids 351, 2616 (2005)

32. G.A. Schwartz, J. Colmenero, A. Alegría, Macromolecules 39, 3931 (2006)

33. G.A. Schwartz, J. Colmenero, A. Alegría, Macromolecules 40, 3246 (2007)

34. G.A. Schwartz, J. Colmenero, A. Alegría, J. Non-Cryst. Solids 353, 4298 (2007)

35. M. Tyagi, J. Colmenero, A. Alegría, J. Chem. Phys. 122, 244909 (2005)

36. D. Hall, P. Underhill, J.M. Torkelson, Polym. Eng. Sci. 38, 2039 (1998)

37. S. Gomez-Monivas, L.S. Froufe, R. Carminati, J.J. Greffet, J.J. Saenz, Nanotechnology 12, 496 (2001)

38. B. Bhushan, H. Fuchs, Applied Scanning Probe Methods II (Springer, 2003), p. 312 